EGU2020-5421, updated on 03 Nov 2021

https://doi.org/10.5194/egusphere-egu2020-5421

EGU General Assembly 2020

(c) Author(s) 2021. This work is distributed under

the Creative Commons Attribution 4.0 License.

\title{
Twelve years of SIFTER Sun-Induced Fluorescence retrievals from GOME-2 as an independent constraint on photosynthesis across continents and biomes
}

Maurits L. Kooreman ${ }^{1}$, K. Folkert Boersma ${ }^{1,2}$, Erik van Schaik ${ }^{2}$, Anteneh G. Mengistu ${ }^{1,3}$, Olaf N. E. Tuinder ${ }^{1}$, Piet Stammes ${ }^{1}$, Gerbrand Koren ${ }^{2}$, and Wouter Peters ${ }^{2}$

${ }^{1}$ Royal Netherlands Meteorological Institute, De Bilt, The Netherlands

${ }^{2}$ Wageningen University and Research, Wageningen, The Netherlands

${ }^{3}$ Addis Ababa University, Addis Ababa, Ethiopia

Solar-Induced Fluorescence (SIF) data from satellites are increasingly used as a proxy for photosynthetic activity by vegetation, and as a constraint on gross primary production. The Royal Netherlands Meteorological Institute has developed an improved retrieval algorithm called SIFTER, to retrieve mid-morning (09:30 hrs local time) SIF estimates on the global scale from GOME-2 sensors on the Metop satellite series. The product is developed within the ACSAF network of EUMETSAT and a beta version is publicly available on www.temis.nl. The SIFTER algorithm improves over a previous version by using a narrower spectral window that avoids strong oxygen absorption and is less sensitive to water vapor absorption, by constructing stable reference spectra from a 6-year period (2007-2012) of atmospheric spectra over the Sahara, and by applying a latitude-dependent zero-level adjustment that accounts for biases in the product data. With SIFTER, we generate stable, good-quality SIF retrievals also in tropical regions that are known to suffer from high noise in other SIF products. Uncertainty estimates are included for individual observations, and the product is best used for mostly clear-sky scenes, and when spectral residuals remain below a certain threshold. The strength of SIFTER in the tropical regions was exploited to quantify the 2015/2016 drought in the Amazon, related to El Niño. We found that SIF was strongly suppressed over areas with anomalously high temperatures and decreased levels of soil moisture. SIF went below its climatological range starting from the end of the 2015 dry season and returned to normal levels by February 2016. A validation study is performed to assess the SIFTER quality against independent SIF and GPP products from other platforms, including SIF from OCO-2 and GOSAT, modeled GPP from MPI-BGC and eddy covariance derived, in-situ GPP measurements. SIFTER shows strong correlations $(0.70-0.94)$ in the zonal distribution for each continent and in capturing seasonal patterns of SIF and GPP over different regions across the globe (0.62-0.99) when comparing to OCO-2 SIF and GPP from MPI-BGC. At ecosystem level, SIFTER was evaluated against OCO-2 SIF and EC GPP for five flux tower sites with varying biomes and geolocations. Regions with a homogeneous vegetation distribution show a higher correlation than heterogeneous regions. Overall, the results support the use of SIFTER data to be used as an independent constraint on photosynthetic activity on global and regional scales. 
